\title{
DISTRIBUTED OPTIMAL CONTROL MODELS IN ENVIRONMENTAL ECONOMICS: A REVIEW
}

\author{
Emmanuelle Augeraud-Véron ${ }^{1, *}$, Raouf BoucekKine ${ }^{2}$ \\ AND VLADIMIR M. VELIOV ${ }^{3}$
}

\begin{abstract}
We review the most recent advances in distributed optimal control applied to Environmental Economics, covering in particular problems where the state dynamics are governed by partial differential equations (PDEs). This is a quite fresh application area of distributed optimal control, which has already suggested several new mathematical research lines due to the specificities of the Environmental Economics problems involved. We enhance the latter through a survey of the variety of themes and associated mathematical structures beared by this literature. We also provide a quick tour of the existing tools in the theory of distributed optimal control that have been applied so far in Environmental Economics.
\end{abstract}

Mathematics Subject Classification. 91B76, 35Q91, 49-02.

Received January 4, 2019. Accepted March 15, 2019.

\section{INTRODUCTION}

\subsection{Aim of the review}

The complexity and the fine structures encountered in Environmental Economics increasingly involve distributed systems, usually in the form of partial differential equations (PDEs).

An excellent review on applications of PDE optimal control to problems of management of environmental resources is presented in the paper by Brock et al. [34]. This review focuses primarily on models described by diffusion-reaction equations. The aim of the present paper is to complement [34] in several aspects. First, we include contributions published after [34], taking into account the large amount of recent work in the field. Second, we extend the scope of the review by including a variety of distributed control models: reactiondiffusion-advection problems, general first order equations with non-local dynamics, age-structured and sizestructured models. Third, in contrast to [34], where the exposition is mainly based on the maximum principle, we attribute more attention to the dynamic programing approach and the associated Hamilton-Jacobi-Bellman (HJB) equation. This approach can be advantageous, especially in the cases where an explicit solution can be obtained.

Keywords and phrases: Environmental economics, distributed systems, optimal control, partial differential equations.

1 Bordeaux University, GREThA (UMR CNRS 5113), Bordeaux, France.

2 Aix-Marseille University (AMSE and IMéRA), IUF, Provence, France.

3 ORCOS, Vienna University of Technology, Wien, Austria.

* Corresponding author: emmanuelle.augeraud@u-bordeaux.fr 
Shortly speaking, in this paper we try to present the diversity of the problems considered in Environmental Economics, indicating first the application areas (Sect. 2), then reviewing several kinds of mathematical structures and problems arising in Environmental Economics (Sect. 3) and solution approaches and instruments (Sect. 4). Along with this review, we shall briefly present the contributions of the papers included in the present special issue.

\subsection{Specific features of the distributed optimal control problems in economics}

\subsubsection{Infinite horizon}

One specific feature of economic models, especially in Environmental Economics, is that the optimal control problems that arise are on an infinite horizon. For such problems, the usual transversality conditions for the adjoint (co-state) function associated with finite-horizon problems have to take the form of asymptotic (transversality) conditions. As known from the famous example by Halkin [55] and numerous later papers, the transition from a finite to an infinite horizon is a delicate matter (the Pontryagin maximum principle may even fail to hold in a normal form - with a non-zero Lagrange multiplier to the objective functional - even without any state constraints). Despite the numerous contributions addressing the issue of asymptotic conditions (e.g. Michel [65]) or other ways to identify the "right" solution of the adjoint equation (e.g. Aseev and Kryazhimskii [11], Aseev and Veliov [12], Trauchnitz [70]), the theory of infinite horizon problems is still incomplete even for problems with ordinary differential equations (ODEs). An additional complication arises in environmental considerations from the fact that the objective functional may take infinite values, especially if the future is not (heavily) discounted due to sustainability arguments. Then one can use the notion of overtaking optimality or another of the numerous notions of optimality which are meaningful in the case of improper objective integral (see e.g. Carlson et al. [38]).

The issue of asymptotic/transversality conditions in distributed optimal control problems becomes substantially more complicated than in the ODE case, being in the same time important in Environmental Economics. We return to this issue in the next section.

\subsubsection{Nonlinearities}

Nonlinearities, encountered in the environmental systems due to complex interactions, can lead to various types of complex behavior of the optimal trajectories, such as periodicity, indeterminacy and thresholds (see e.g. Grass et al. [52]). There is a huge amount of literature on these phenomena for ODE-problems, based on bifurcation analysis and analysis of the stable invariant manifold of the associated state-adjoint system.

This is not the case for distributed optimal control problems, where only a few papers report results about global indeterminacy and thresholds: Feichtinger et al. [48] in a distributed epidemiological model, Grass and Uetcker [53] for a distributed pollution model of shallow lakes.

\section{Control of Distributed Systems in ECONOMiCS}

In this section, we present various fields of application of distributed optimal control problems in Environmental Economics.

\subsection{Pollution}

The optimal management of pollution has been studied in the context of spatial models for a long time. The nature of the distributed system considered ultimately depends on the nature of the physical characteristics of pollution. In the case of atmospheric pollution, or even in the case of pollution of shallow lakes (Grass and Uecker [53]), the major phenomenon involved is diffusion. Camacho and Pérez-Barahona [36] studied the problem of atmospheric transboundary pollution in the context of an optimal land use problem, with a finite optimization time horizon. In this issue, Boucekkine et al. [28] abstract away from the latter optimal land use problem 
and focus on a production-induced pollution social planner problem with trans-boundary diffusive pollution, negative environmental externalities and space-dependent environmental awareness across population. They also consider a framework with an infinite time horizon, which in principle opens the door for a deep analysis of the asymptotic properties, and in particular space dependence of the possible stationary solutions. Indeed, the linear-quadratic setting used allows to extract closed-form solutions either for the optimal dynamics or for the asymptotic spatial distributions.

The case of groundwater, and more generally of diffusive pollution subject to a particular directional transport (current, prevailing wind), requires the consideration of a diffusion-convection type of spatial dynamics. De Frutos and Martín-Herrán [41] study the problem of transporting a pollutant subject to a river current as part of a game between different cross-border entities. The game is then studied in a discrete space framework, considering each cross border entity as a single patch. Comparison of the spatial and non-spatial solutions in both a cooperative and non-cooperative setting is undertaken in a companion paper by De Frutos and MartínHerrán [42]. They show in particular that the spatial model is a generalization of the model without the spatial aspects in the non-cooperative Nash case: as the pollution diffusion parameter tends to infinity, the equilibrium feedback policies converge to those of the non-spatial setting. Augeraud-Véron et al. [13] consider a problem of nitrate pollution in groundwater including a detailed modeling of the transport of the pollutant using the hydro-geological properties of the environment. In particular, a nonlinearity is considered at the diffusion level, in order to take into account the fact that the dispersion coefficient depends nonlinearly on the speed of pollution particles. The existence of a solution of the inherent optimal control problem is proved. Within the same framework, it is shown in Choquet et al. [39] that the uniqueness of the solution is ensured in the case of low pollution concentration, as it is the case for nitrate pollution.

\subsection{Harvesting}

Distributed models for optimal harvesting appear mainly due to two reasons:

(i) The bio-resources are often situated in large areas and their distribution is not homogeneous. The resource can move in the space due to diffusion or migration, as in fishery in open basins, or have a permanent residence, as in forestry, where the heterogeneity appears due to differences in the time of planting or in the growth rate.

(ii) The age- or the size-distribution of bio-resource plays an important role both in fishery and in forestry.

In distributed optimal harvesting problems, the control (the harvesting effort is always a control variable, but possibly also fertilizing and watering efforts in agriculture can be regarded as controls, among others) can be either distributed or only time-dependent.

From the literature on optimal harvesting in age-structured populations (see Tahvonen's review paper [69]) we mention Aniţa et al. [6, 10] and Luo et al. [63], where the model parameters such as vital rates and inflow forcing terms may be periodic in time due to exogenous (seasonal) changes. A general size-structured population model with periodic parameters is investigated in He and Liu [56]. In these papers the optimal controls are also (asymptotically) periodic, which is a result of periodicity of the data. In Belyakov and Veliov [21, 22], however, it is shown that periodic optimal harvesting may appear if the age-structure is taken into account in a singlespecies model with natural off-spring production and constant parameters, both in the case of maximization of the total discounted net revenue, and of the averaged net revenue. Complementary results in the same spirit are given in the recent paper by Golubtsov and Steinshamn [51]. We mention that periodic optimal control policies are well-known in the forestry and timber production, and also for Lotka-Volterra type ODE-models with multiple species (pray-predator). The selective industrial fishing is known to create huge detrimental genetic and phenotypic effects (e.g. Landi et al. [60]). A policy consisting of periods of intensive harvesting followed by recovery periods might be more productive in both ecological and economic sense, compared with the usual persistent harvesting at a modest rate. However, to the authors' knowledge, optimal control models involving the genetic parameters have not been investigated. 
A review of the literature on harvesting of spatially distributed resource is given in Brock et al. [34]. This literature has increased a lot since then. For example, one can refer to Braack et al. [30] who consider an optimal harvesting control problem where the discounted present value of profits plus the remaining fish stock at final time is maximized. Besides the existence of an optimal solution which is proved in this non-convex optimal control problem, numerical solutions are computed. The authors draw a parallel between the regions where the optimal solution is not to harvest and the regions where No-Take-Zones and maximal fishing quotas should be applied. We extend Brock et al.'s review, mentioning two additional research directions.

The first direction addresses the problem of optimal allocation of harvesting in a "small" sub-domain of the domain occupied by the resource. Kann et al. [58], for example, investigate a spatial model of coastal fishing. Aniţa et al. [5] consider a profit maximization problem on a finite interval, where the fishing effort is a control and the growth dynamics follows the logistic low. Although the resource can move in a relatively large open area, the fishing areas are on the contrary, confined to small sub-areas. Existence of an optimal control is proved in this case, and the optimal spatio-temporal distribution of the fishing effort is obtained numerically by employing the Pontryagin maximum principle. Structuring in space also makes it possible to consider fisheries that only operate along the boundary of a basin, as it is the case for coastal fisheries. In this context, Grass et al. [54] consider a problem of maximizing profit over an infinite horizon. The problem of optimal choice of this "small" sub-domain was recently investigated in Aniţa et al. [8]. The question of the shape of the fishing domain is tackled with another focus, in Joshi et al. [57], where Dirichlet boundary conditions are considered, such that the boundary of the domain is lethal for fish. In Kelly et al. [59], the same model is considered, with Robin boundary conditions, and the impact of the boundary conditions on the optimal solution is emphasized.

The second direction is about problems of finding the optimal mode of motion of the harvester in the space. The harvesting rate at any place in the space depends on (among other factors) the mode of motion (velocity at that place, frequency of harvesting at the same place, etc.). There are various particular formulations, but the mathematical problems that arise are usually non-standard; see e.g. $[18,20]$.

The paper by Aniţa et al. [7] included in this issue builds on Belyakov et al. [20] and Behringer and Upmann [18] by introducing in the model endogenous market demand, which may lead (in contrast to [18]) to only partial extraction of the harvested resource.

\subsection{Migration}

The fundamental work by McKendrick (1926) introduces the age-structured models with non-local boundary conditions in the context of demography. Optimal migration patterns can also be investigated using that kind of models and their numerous extensions. Simon et al. [67], for example, focus on the optimal age-structure of migrants in a population with constant size, regarding economic objectives. In the present issue, Camacho and Pérez-Barahona [37] consider spatial issues. The pure economic literature on migration has been mostly concerned with static models (we abstract here from 2-country models which do not really attempt to uncover specific geographic patterns). Perhaps the most impressive piece of work in this stream, both in terms of technical value and economic micro-foundation, is due to Mossay [66], who tried to go beyond the typical wage gradient to motivate migrations, so common in the 2-country modeling, by considering idiosyncratic local migrations in the continuous space. In the subsequent New Economic Geography literature, attempts to devise dynamic models of migration with spatial ingredients are rather scarce, especially in continuous space (see an overview in Fujita and Thisse [50]).

In this sense, Camacho and Pérez-Barahona [37], selected in this special issue, is a valuable contribution. Indeed, the idea to model migration as a diffusion process of human capital is far from inaccurate. It is widely admitted that skilled individuals are more likely to migrate (again see Fujita and Thisse, for example Chap. 11 and references therein). Of course, such a modeling cannot capture all the heterogeneity embodied in the migration phenomenon but still, as demonstrated in the numerical simulations provided by Camacho and PérezBarahona, it is enough to generate a rich set of migration spatial patterns. In particular, the role of barriers to migration is underlined and the realism of the generated migration patterns discussed. 
Also in this issue, La Torre et al. [61] consider a problem of population dynamics in a spatial context. The authors endogenize both mortality and fertility at any location, and allow for migrations through locations via a diffusion process as in Camacho and Pérez-Barahona [37]. A key control variable is the fraction of time devoted to productive activities vs. rising children at any location, which founds endogenous fertility in the setting. The endogeneity of mortality derives instead from the following standard mechanism (see for example Mariani et al. [64]): the higher the density of population on a given location, the larger pollution and subsequently the lower the local survival conditions. The authors compare numerically the solutions under central planning and those obtained in a decentralized framework, ultimately showing the fundamental role of spatial externalities.

\subsection{Land use}

Utilization of land is a broad thematic that concerns many kind of problems, including ecology, geography and economics. Uecker [72] considers a spatial semi-arid land use problem to optimize livestock farming. Livestock eats the vegetation, which regenerates slowly under the effect of rare rain falls. In particular, it is shown that taking spatially distributed control into account delivers much more accurate and therefore more useful results than if the control depends only on time. The numerical simulation of the optimality conditions obtained via the application of the Pontryagin maximum principle shows the existence of optimal harvesting patterns, and highlights incidentally the strong dependency of the asymptotic optimal control on space.

Camacho and Pérez-Barahona [36] is one of the very first papers in the economic literature taking the challenge of studying optimal land use in spatio-temporal model with environmental degradation and transboundary pollution. This is a major topic in agricultural research, for example, but the related studies are largely empirical (see for example Adams et al. [1]). In Camacho and Pérez-Barahona, each location is endowed with a fixed amount of land, which is allocated among production, pollution abatement and housing. Although the unique production input (land) is spatially immobile by nature, this is a model of spatial growth where locations actions affect the entire space through pollution, which is governed by a diffusion equation. The authors compute the optimality conditions corresponding to a social planner problem with a finite time horizon and land (and population) distributed along the real line. Numerical simulations of discretized versions of the Pontryagin conditions deliver various interesting results. A particularly important result has to do with the role of global versus local pollution on the optimal spatial pattern. One might think that global pollution is homogenizing. However, Camacho and Pérez-Barahona show that spatial heterogeneity can emerge even when pollution only has global effects, due to pollution diffusion and the spatial specificity of abatement activities.

\subsection{Spatial growth models}

While this review is concerned with environmental modeling, it is worth mentioning the strong nexus with the contemporaneous economic literature on spatial economic growth models. Typically, the latter involves the optimal control of production factors' spatio-temporal dynamics governed by a diffusion equation (see Brito [32], for the first attempt, and Boucekkine et al. [29] for the ultimate one). Therefore, the two classes of models involve similar distributed control problems. For example, Boucekkine et al. [25] consider in particular an AK growth model with capital diffusion for which they explicitly calculate the optimal control using the HJB method. They show in particular that the optimal control is independent of space. This same problem is studied using the Pontryagin maximum principle by Ballestra [15] (see also Boucekkine et al. [26]). Last but not least, Fabbri [43] has specifically studied the impact of the shape of the domain on the optimal solution in this class of spatial growth models. In this issue, Augeraud-Véron and Ducrot [14] assess the impact of inter-regional disparities when the engine of growth is a learning by doing externality, modeled through non-local interactions. It is in particular shown that, depending on the type of interaction considered, local indeterminacy may occur. Also in this issue, Boucekkine et al. [28] consider a special case of the geographic AK growth model referred to above, with full capital depreciation in order to focus on a single diffusion equation (transboundary pollution). 


\section{Mathematical Structures and problems in EnVironmental ECONOMICS}

\subsection{Non-local equations}

Non-local interactions are typical in distributed models in Environmental Economics in which agents (in broad sense) may influence the other agents behavior even if they are not necessarily close to each other in the "spatial" domain. For many kinds of agents these interactions are usually expressed by aggregated quantities, often appearing as externalities, in economic terms. For example, the total output of an industry branch consisting of non-monopolistic firms is an aggregated quantity that influences the individual firms' behavior through the market prices. In models involving populations, the total (possibly weighted) size of the population or the total biomass influence the amount of newborns, and for wild animal populations also the mortality rates.

Another type of interactions, different from the aggregated ones, appears if the agent's behavior is influenced by the state and control of another agent depending on a certain "distance" (in a non-strict sense) between them. For example, the contact rate of an individual of a certain age with other individuals in a population depends on the difference between the ages; the growth rate of a tree depends on the sizes of the trees around it more than on the sizes of distant trees, etc.

The state equation of a control model involving distant interactions can often be written in the form of a coupled system of ODEs, parameterized by a parameter $\sigma$ belonging to a measurable set $\Sigma$ :

$$
\dot{y}(t, \sigma)=f(y(t, \sigma), z(t, \sigma), u(t, \sigma), v(t))
$$

where $y(t, \sigma)$ is the distributed state at time $t$. The parameter $\sigma$ can be regarded as representing specific individual features, therefore it is often called parameter of heterogeneity, and the set $\Sigma$ is the domain of heterogeneity. The function $u(t, \sigma)$ is a distributed control variable and $v(t)$ is a non-distributed control variable. Then both types of interactions mentioned in the last paragraph can be represented by the term $z$ in (3.1) having the form

$$
z(t, \sigma)=\int_{\Sigma} g\left(t, \sigma^{\prime}, \sigma\right) y\left(t, \sigma^{\prime}\right) \mathrm{d} \sigma^{\prime}
$$

If the kernel $g$ is independent of $\sigma$, then $z=z(t)$ is also independent of $\sigma$, thus it is just a weighted aggregated variable. Often $g$ has the form $g\left(\sigma^{\prime}, \sigma\right)=g\left(\sigma^{\prime}-\sigma\right)$ (as in the examples mentioned at the end of the last paragraph). In this case, we encounter convolution type of non-local variable $z(t, \sigma)$ :

$$
z(t, \sigma)=\int_{\Sigma} g\left(t, \sigma^{\prime}-\sigma\right) y\left(t, \sigma^{\prime}\right) \mathrm{d} \sigma^{\prime}
$$

Necessary optimality conditions of Pontryagin's type for a general optimal control problems of the kind of (3.1), (3.2) are obtained in Veliov [73], where the objective functional contains a terminal term taking account agents who are still alive at the terminal date, denoted by $\Omega(T)$, and an intertemporal term summing the current "revenue" over the time and the leaving agents at each time:

$$
\int_{\Omega(T)} l(\sigma, x(T, \sigma)) \mathrm{d} \sigma+\int_{0}^{T} \int_{\Omega(t)} L(t, \sigma, x(t, \sigma), y(t), u(t, \sigma), v(t)) \mathrm{d} \sigma \mathrm{d} t .
$$

We mention that non-local dynamics also appears in models with diffusion, where the spatial domain represents a physical region. This is the case in Aniţa et al. [9], where the non-local term like in (3.2) appears in the logistic low in the dynamics of a space-distributed population. 


\subsection{Age/size-structured models}

A typical representative of the age-structured optimal control problems (with the dynamics described by the original McHendrick equation) is the one of maximization of the functional

$$
\int_{0}^{T} \int_{0}^{A} L(t, y(a, t), u(a, t), a) \mathrm{d} a \mathrm{~d} t
$$

subject to the equation

$$
\left(\frac{\partial}{\partial t}+\frac{\partial}{\partial a}\right) y(a, t)=-\mu(a, t) y(a, t)+u(a, t) f(a, t)
$$

with initial and boundary conditions

$$
y(a, 0)=y_{0}(a), \quad y(0, t)=z(t),
$$

and control constraints $u(t, a) \in[a, b]$, where $z(t)$ is an aggregated non-local variable representing the newborn population:

$$
z(t)=\int_{0}^{A} \beta(a, t) y(a, t) \mathrm{d} a
$$

Clearly, here $\mu$ represents the mortality rate, $\beta$ is the fertility rate, both depending on the age $a \in[0, A]$. We use the traditional notation $\left(\frac{\partial}{\partial t}+\frac{\partial}{\partial a}\right) y$ for the directional derivative of $y$ in the direction $(1,1)$, although the both partial derivatives may fail to exist.

Numerous extensions arising in population and economic modeling are known: nonlinear right-hand side of the differential equation, possibly dependent on non-local variables, nonlinear dependence of the non-local variables on the state function, non-distributed control $v(t)$ that may also enter in the boundary conditions, etc. Another extension concerns the differential operator in the left-hand-side of the differential equation, which can take the form $\frac{\partial y}{\partial t}+\frac{\partial(p(t, a, z(t)) y)}{\partial a}$, in which case $a$ is interpreted as size rather than age, and $p(t, a, z(t))$ is the growth rate at size $a$, which may depend on the time and on some aggregated non-local variables.

All the models of the dynamics in this subsection are particular cases of the heterogeneous model (3.1), (3.2) furnished with appropriate initial and boundary conditions, and with an appropriate domain of heterogeneity (see Veliov [73]).

General necessary optimality conditions of Pontryagin's type for optimal control problems for age-structured systems are first obtained in Brokate [35] in the case of aggregated variables $z(t)$ (that is, $g$ is independent of $\sigma$ in Eq. (3.2)). Pontryagin's type maximum principle is obtained for more general age-structured problems in Feichtinger et al. [47]. In both papers the time horizon is finite. The case of infinite horizon is investigated in Skritek and Veliov [68].

\subsection{Space-structured models}

A majority of spatially structured models are of the reaction-diffusion form, where the reaction process allows to describe the biological, chemical, etc. phenomena to which the state variables are subjected and where the diffusion reflects the often iso-spatial dispersion of the state variable. These phenomena are described using parabolic partial differential equations

$$
\frac{\partial}{\partial t} y(x, t)-D \Delta y(x, t),=f(y(x, t), u(x, t), x)
$$


where boundary conditions $(x, t) \in \partial \Omega \times(0, T)$ may be given (if the spatial domain of interest, $\Omega$, is not all the space $\mathbb{R}$ ) and an initial condition $y(x, 0)=y_{0}(x)$ is given. In equation (3.4), $D$ is a differential operator (with respect to the spatial variable $x$ ) capturing diffusion, and $f$ is the reaction function, which depends on the state $y$ of the system and on the control variable $u$. The time horizon $[0, T]$ can be finite or infinite. For some models, such as hydro geological models, the transport of a pollutant may require a diffusion that depends on the state of the system, so that the diffusion term may take the form $\operatorname{div}(\eta(y(x, t)) \nabla y(x, t))$. An additional term of the form $\nabla(\sigma(x, t) y(x, t))$ may be present in (3.4), representing advection with velocity field $\sigma$.

Optimal control problems for parabolic systems can be written as

$$
\max _{u(x, t) \in U} \int_{0}^{T} e^{-\rho t} \int_{\omega} g\left(u(x, t), y^{u}(x, t), x\right) \mathrm{d} x \mathrm{~d} t,
$$

subject to point-wise control constraints, $u(x, t) \in U$, where $U$ is a given set and $y^{u}(a, t)$ is the solution of (3.4) corresponding to $u$, with given boundary conditions (if needed) and an initial condition. Usually the spatial domain $\Omega$ is a bounded open set in a finite-dimensional space, and $\omega$ is a nonempty subset of $\Omega$.

This type of problems has been studied, in particular, by Aniţa et al. [5], where the time horizon is finite and the growth law of the state $y$ (interpreted as a resource stock) is given by a logistic function. The instantaneous yield is proportional to $u(x, t) y(x, t)$. Existence and uniqueness of optimal solution is proved and a Pontryagintype maximum principle is obtained. Grass and Uecker [53] consider a pollution problem where the reaction function is a combination of a linear function and a function of the form $\frac{y^{2}}{1+y^{2}}$ (a particular case of the so called Sips functions, see e.g. Belhachemi and Adoun [19]). Numerical simulations are done to study bifurcations and existence of patterned steady states. Boucekkine et al. [28] consider a linear reaction function with a quadratic linear objective, in order to obtain closed form solutions.

Aniţa et al. [9] consider parabolic models involving an additional "spatial" variable (interpreted as age), thus incorporating the age-structure (of a harvested/utilized resource, for example) into the spatial dynamics. Models of this kind are important in the case of biological resources, but also for resource in the form of physical capital stocks, because such resources have its own life-cycle dynamics which may have substantial impact on the overall dynamics involved in the modeling.

If the spatial domain $\Omega$ is the whole space, then the model (3.4) does not require boundary conditions. This is also the case when $\Omega$ is a compact, connected, oriented Riemannian manifold without boundary (see Fabbri [43]).

In the case of models involving a bounded spatial domain $\Omega$, the boundary conditions are usually of three kinds:

(i) Dirichlet-type conditions, $y(x, t)=y_{1}(x, t)$ a.e. $(x, t) \in \partial \Omega \times(0, T)$, with a given $y_{1}(x, t) \in L^{\infty}$, relevant if the state at the boundary is exogenously prescribed and known;

(ii) Neumann type conditions $\nabla_{\nu} y(x, t)=y_{1}(x, t)$ a.e. $(x, t) \in \partial \Omega \times(0, T)$ (here $\nabla_{\nu} y(x, t)$ is the directional derivative in the direction of the outward unit normal vector $\nu(x)$ to $\Omega$ at $x$ ), where the flux $y_{1}(x, t)$ at the boundary of the domain is known;

(iii) Robin type conditions, $\nabla_{\nu} y(x, t)+\delta y(x, t)=0$ if, in particular, the inflow is proportional to a jump between two media.

The control can appear not only in the equation of the dynamics (i.e. in (3.4)), but also in the boundary conditions. This is often the case of PDE-constraint optimization, in general, but also appears in problems related to the environment, say in case of coastal fishery. In Grass et al. [54], boundary control appears when modeling a problem of pollution control on the shore of a lake, where the pollutant is diffusing into the environment. 


\section{InVESTIGATION TOOLS}

\subsection{Existence}

Only a few works on distributed optimal control problems related to Environmental Economics address the issue of existence of optimal solutions. As for every minimization problem, the existence issue consists of three components:

(i) existence of feasible control-trajectory pairs;

(ii) boundedness (from below) of the objective functional on the feasible set;

(iii) existence of a minimizer.

The first component includes choosing appropriate spaces for the control and the state functions, in order to ensure existence of a solution of the underlying differential equations. The choice of spaces very much depends on the problem at hand and the (expected) regularity of the solutions. The issue is comprehensively elaborated in the PDE literature, see e.g. Fattorini [46] or Li and Yong [62] in the optimal control context. Different in nature and more demanding is to prove existence of control-trajectory pairs satisfying additional constraints for the state function, such as non-negativity (as it often appears in economics) or terminal constraints. Such proofs usually relay on specific features of the problem at hand.

The common approach to component (iii), given that feasible control-trajectory pairs exist, is the one introduced by Tonelli in calculus of variations: to ensure existence of a minimizing sequence that is precompact in a topology in which one can take the limit in the constraints, and in which the objective functional is lower semi-continuous. The choice of the Hilbert space $L^{2}$ for the controls allows for using the weak topology (and the Banach-Alaoglu weak compactness theorem), provided that a bounded minimizing sequence exists. Then convexity in the control of the objective functional and linearity in the control of the equations make it possible to pass to a limit. Here, the Mazur-Banach theorem plays a substantial role in the proofs, since it allows to produce another control sequence (consisting of convex combinations of the elements of the minimizing control sequence), which is strongly convergent to the same limit.

If the equations are non-linear in the control (and the set of admissible "velocities" is non-convex), one can use the convexification technique for passing to a relaxed problem to which the Tonelli approach can be applied, see e.g. Fattorini (Part III of [46]).

Sometimes the space $L^{1}$ is used for the controls, in which case the Dunford-Pettis criterion for weak compactness can be used. Alternatively, one can first prove existence in the space $L^{2} \subset L^{1}$, then use the uniqueness of the weak limit and the dominated convergence theorem in $L^{1}$ to prove the existence of the solution in $L^{1}$ (see e.g. Aniţa [5]). Coclite and Garavello [40] consider the existence of solutions in an optimal harvesting problem with linear control. In such a case, there is no guarantee that the optimal solution belongs to $L^{1}$. Therefore, the authors consider the problem in the space of bounded Radon measures. In this sense, they bring out a new insight into the problem tackled in Bressan et al. [31], using a similar but stationary model.

For problems with non-local dynamics, especially for age-structured problems, one may need to first apply the Dunford-Pettis theorem or the Banach-Alaoglu theorem to the state functions in a minimizing sequence, then to pass to a new sequence of state functions using the Mazur-Banach theorem, and after that to construct a sequence of controls producing these state functions via the differential equation. This approach originates from [10] and although it is only applicable to problems with a rather special structure, it was successfully modified and extended in Feichtinger and Veliov [49] and Simon et al. [67].

The situation where the objective functional is not semi-continuous can sometimes be circumvented by applying the Ekelend variational principle, see e.g. Barbu and Iannelli [16], for a population control problem.

One way of proving boundedness from below of the objective functional (issue (ii) mentioned above) is to apply comparison theorems, which is possible in many parabolic problems, see Chapter 3 of Li and Yong [62]. However, for complicated problems involving non-linearities (in the reaction function, the diffusion coefficients, etc.) the comparison theorems are difficult to apply. Moreover, these nonlinearities make it impossible to use convex combinations of controls for proving existence making use of the Mazur-Banach theorem. In that case, 
Schauder's fixed point theorem can be applied to prove existence of an optimal solution in finite horizon problems (see for instance Augeraud et al. [13]). The extension to optimal problems with infinite planning horizon can be done using Amann's theorem [2,3].

\subsection{Maximum principle}

A main tool for qualitative analysis and for developing numerical methods for distributed optimal control problems is provided by various extensions of the classical Pontryagin maximum principle. We refer to the books $[46,62,71]$ for formulations and derivations of maximum principles, as first order necessary optimality conditions, for various optimal control problems with PDE-constraints. Complemented with appropriate assumptions or additional conditions, the maximum principle may also become a sufficient optimality condition. In particular, the maximum principle is widely used in the literature on Environmental Economics involving distributed optimal control models (we refer again to Brock et al. [34] and the reference therein).

The maximum principle for problem (3.5)-(3.4) in a bounded domain $\Omega$, a finite horizon $[0, T]$, initial conditions $y(x, 0)=y_{0}(x)$, and the boundary condition $\nabla_{\nu} y(x, t)+\delta y(x, t)=0$ for $x \in \partial \Omega \times(0, T)$ reads as

$$
H(x, \hat{y}(x, t), \hat{u}(x, t), \hat{\lambda}(x, t))=\max _{u \in U} H(x, \hat{y}(x, t), u, \hat{\lambda}(x, t)), \quad(x, t) \in \omega \times(0, T),
$$

where $H(x, y, u, \lambda):=g(u, y, x)+\lambda f(u, y, x)$ (called often Hamiltonian), $\hat{u}$ is a feasible control, $\hat{y}$ is the solution of (3.4) (with the specified initial and boundary conditions) for $\hat{u}$, and $\hat{\lambda}$ is the solution of the following adjoint system:

$$
\begin{aligned}
\frac{\partial}{\partial t} \lambda(x, t)+D \Delta \lambda(x, t)= & r \lambda(x, t)-\nabla_{y} f(\hat{y}(x, t), \hat{u}(x, t), x) \lambda(x, t)-\nabla_{y} g(\hat{y}(x, t), \hat{u}(x, t), x), \\
& \nabla_{\nu} \lambda(x, t)+\delta \lambda(x, t)=0, \quad(x, t) \in \partial \Omega \times(0, T), \\
& \lambda(x, T)=0, \quad x \in \Omega .
\end{aligned}
$$

Special attention has to be attributed to the choice of the functional spaces in which the control functions $u$, the state functions $y$, and the corresponding adjoint functions $\lambda$ do belong. Each of the spaces $L^{\infty}(\Omega \times[0, T))$ and $L^{2}(\Omega \times[0, T))$ used for $u$ in the literature has advantages: the first when differentiation with respect to $u$ is involved in the analysis, the second, if existence of a solution is investigated or second order optimality conditions are involved. If the constraining set $U$ for $u$ is compact, then both spaces can be conveniently used. The choice of an appropriate space for the state function $y$ is more sophisticated. The diffusion in (3.4) has a smoothing effect on the solution $y$, so that $y$ and $\lambda$ can be considered as elements of spaces like $L^{2}\left((0, T) ; H^{2}(\Omega)\right)$ with $y_{t}, \lambda_{t} \in L^{2}\left((0, T) ; L^{2}(\Omega)\right)$. The situation is different for first order PDEs like the age-structured equation (3.3). There, the solution $y$ may be even discontinuous with respect to each of the arguments $t$ and $a$, but is absolutely continuous along every (characteristic) line $t-a=$ const. This requires a special choice of the functional space for $y$ (see e.g. Feichtinger et al. [47] and Webb [74]).

Notice that for parabolic problems the adjoint equation contains an anti-diffusive term (with a positive sign in front of the diffusion term). On the other hand, the initial condition is replaced with an end-point condition for $\lambda$, so that the adjoint system is parabolic again.

For infinite horizon problems similarly difficulties as in the case of ODEs, mentioned in Section 1.2.1, appear in the distributed case: one needs asymptotic conditions to complement the adjoint equation with an "end-point" condition. Although terminal constraints on the state are not present, the normal form of the maximum principle may fail. That is, one should consider the possibly abnormal form of the Hamiltonian, $H\left(x, y, u, \lambda, \lambda^{0}\right):=\lambda^{0} g(u, y, x)+\lambda f(u, y, x)$, where $\lambda^{0}$ may have to be taken equal to zero. Another trouble is the need to consider possibly unbounded objective integrals (infinite optimal value) which arises in economic models and in sustainability issues. In practice, standard asymptotic conditions, such as $\lim _{t \rightarrow \infty}\left\langle\lambda, x^{*}\right\rangle_{L^{2}}=0$ (see e.g. Brock and Xeppapadeas [33]), work in most of the considerations. An alternative approach is to build 
on the papers Aseev et al. [11, 12], where the "right" adjoint function is explicitly specified in the ODE case. A step in this direction is the result in Skriteck and Veliov [68] for age-structured problems on infinite horizon.

\subsection{Hamilton-Jacobi-Bellman equation}

Another analytical tool, also used in this issue (see Boucekkine et al. [28]), is the dynamic programming approach as advocated by Barucci and Gozzi [17] and Fabbri and Gozzi [44] in different infinite-dimensional optimal control problems (with state equations governed by first-order PDEs and delay differential equations, respectively). See also a more recent application to the optimal population size problem by Boucekkine et al. [27]. In all these cases, one has first to rewrite the original infinite-dimensional problem as an optimal control problem driven by an ODE in a suitable functional (Hilbert) space, following Bensoussan et al. [24]. This is far from trivial, the main difficulty at this stage is to define the new state variable (usually called structural state) in order to write down the new (ODE) state equation that it satisfies in the functional space. Once this done, the dynamic programming tool is applied, which requires in particular writing and solving the corresponding HJB equation in the functional space. Here comes the second difficulty, a much more widely known difficulty: solving the HJB equation in the Hilbert space. However, as shown for example in Fabbri and Gozzi [44] in a delay differential state equation context or in Boucekkine et al. in this issue for the optimal control of diffusion equations, a number of problems can be solved by obtaining closed form solutions to the HJB. Not surprisingly, these favorable cases cover the typical linear quadratic problems, or economic optimal growth problems which are linear in the state variables and have power instantaneous utility. In such a case, one can identify closedform solutions for the value functions, which in turn delivers the optimal feedbacks. Last, one has eventually to translate back the solution to the original infinite-dimensional setting and write down the corresponding optimal controls, among others.

To give a quick hint into the functioning of this method, at least for the first critical step, consider the optimal control of a diffusion equation. In such a case, a linear operator associated with the diffusion is considered

$$
\begin{array}{r}
\mathcal{A}: D(\mathcal{A}) \subset \mathrm{L}^{2}(\Omega) \rightarrow \mathrm{L}^{2}(\Omega) \\
\mathcal{A} \varphi=\Delta \varphi .
\end{array}
$$

Domain $D(\mathcal{A})$ is a part of Sobolev space $\mathrm{H}^{2}(\Omega)$. For problems written on a compact space without boundaries (Fabbri [43]) or problem written on all $\mathbb{R}^{n}$ (Boucekkine et al. [28]), $D(\mathcal{A})=\mathrm{H}^{2}(\Omega)$. Usual inner product is defined, and, the use of double integration by part enables to compute the adjoint $\mathcal{A}^{*}$ of $\mathcal{A}$. It can be seen that in problems with no boundary, the operator is self-adjoint.

The use of the infinitesimal generator enables to describe the dynamics of the state as an ODE on Hilbert space $\mathrm{L}^{2}(\Omega)$, and to reformulate the objective in abstract form on this space.

The HJB equation then applies to the value function, defined for any initial condition $y_{0} \in \mathrm{L}^{2}(\Omega)$. Taking into account the adjoint operator $\mathcal{A}^{*}$ enables to rewrite the HJB equation in a suitable way.

Two final comments are worth doing here. First of all, it is important to note that all the papers cited above taking the dynamic programming approach do consider infinite time horizon problems, typically because they are concerned with long-term economic growth or sustainable growth themes. Incidentally, infinite-dimensional optimal control problem using HJB have been early used (a survey of the methods can be find in Chapter 6 of Li and Yong [62] for optimal control on finite-horizon. One of the main ingredients that enables to transfer these methods to infinite horizon problem ([43] or [28] for example) is the finiteness of the objective. Second, not surprisingly, these methods can be extended to stochastic frameworks. See the recent book by Fabbri and Gozzi [45] which includes plenty of related abstract problems and applications.

Acknowledgements. The third author is supported by the Austrian Science Foundation (FWF) under grant No P31400N32. 


\section{REFERENCES}

[1] R. Adams, S. Hamilton and B. McCarl, The benefits of pollution control: the case of ozone and U.S. agriculture. Am. J. Agric. Econ. 68 (1986) 886-893

[2] H. Amann, Dynamic theory of quasilinear parabolic systems. Math. Z. 202 (1989) 219-250

[3] H. Amann, Dynamic theory of quasilinear parabolic systems. Math. Z. 205 (1990) 331-331

[4] S. Aniţa and V. Arnautu, Some aspects concerning the optimal harvesting problem. Sci. Annals Univ. "Ion Ionescu de la Brad" Iatsi 48 (2005) 65-73

[5] S. Aniţa, V. Arnautu and V. Capasso, An Introduction to Optimal Control Problems in Life Sciences and Economics, From Mathematical Models to Numerical Simulation with MATLAB, Modeling and Simulation in Science, Engineering and Technology. Birkhäuser, Boston (2010)

[6] S. Aniţa, V. Arnăutu and R. Ştefănescu, Numerical optimal harvesting for a periodic age-structured population dynamics with logistic term. Numer. Funct. Anal. Optim. 30 (2009) 183-198

[7] S. Anița, S. Behringer, A.M. Mosneagu and T. Upmann, Optimal harvesting of a spatially distributed renewable resource with endogenous pricing. In: Special issue Economics and the environment: distributed optimal control models. MMNP 14 (2019) 101

[8] L.I. Aniţa, V. Capasso and A.M. Mosneagu, Optimal harvesting for periodic age-dependent population dynamics. SIAM J. Appl. Math. 58 (1998) 1648-1666

[9] L.I. Aniţa, V. Capasso and A.M. Mosneagu, Regional control in optimal harvesting of population dynamics. Nonlinear Anal. 147 (2016) 191-212

[10] L.I. Aniţa, M. Iannelli, M.Y. Kim and E.J. Park, Optimal harvesting for periodic age-dependent population dynamics. SIAM J. Appl. Math. 58 (1998) 1648-1666

[11] S.M. Aseev and A.V. Kryazhimskii, The Pontryagin maximum principle and transversality conditions for a class optimal control problems with infinite time horizons. SIAM J. Control Optim. 43 (2004) 1094-1119

[12] S. Aseev and V. Veliov, Maximum principle for infinite-horizon optimal control problems under weak regularity assumptions. Proc. Steklov Inst. Math. 291 (2015) 22-39

[13] E. Augeraud-Véron, C. Choquet and É. Comte, Optimal control for a groundwater pollution ruled by a convection-diffusionreaction problem. J. Optim. Theory Appl. 173 (2017) 941-966

[14] E. Augeraud-Véron and A. Ducrot, Spatial externality and intederminacy. In: Special issue Economics and the environment: distributed optimal control models. MMNP 14 (2019) 102

[15] L.V. Ballestra, The spatial AK model and the Pontryagin maximum principle. J. Math. Econ. 67 (2016) 87-94

[16] V. Barbu and M. Iannelli, Optimal control of population dynamics. J. Optim. Theory Appl. 102 (1999) 1-14

[17] E. Barucci and F. Gozzi, Technology adoption and accumulation in a vintage-capital model. J. Econ. 74 (2001) 1-38

[18] S. Behringer and T. Upmann, Optimal harvesting of a spatial renewable resource. J. Econ. Dyn. Control 42 (2014) $105-120$

[19] M. Belhachemi and F. Addoun, Comparative adsorption isotherms and modeling of methylene blue onto activated carbons. Appl. Water Sci. 1 (2011) 111-117

[20] A.O. Belyakov, A.A. Davydov and V.M. Veliov, Optimal cyclic exploitation of renewable resources. J. Dyn. Control Syst. 21 (2015) 475-494

[21] A. Belyakov and V.M. Veliov, Constant versus periodic fishing: age structured optimal control approach. MMNP 9 (2014) $20-38$

[22] A.O. Belyakov and V.M. Veliov, On optimal harvesting in age-structured populations, in Dynamic Perspectives on Managerial Decision Making - Essays in Honor of Richard Hartl, edited by H. Dawid et al. Springer, Switzerland (2016) 149-166

[23] C. Benosman, B. Aïnseba and A. Ducrot, Optimization of cytostatic leukemia therapy in an advection-reaction-diffusion model. J. Optim. Theory Appl. 167 (2014) 296-325

[24] A. Bensoussan, G. Da Prato, M.C. Delfour and S.K. Mitter, Representation and Control of Infinite Dimensional Systems. Springer Science and Business Media, Berlin (2007)

[25] R. Boucekkine, C. Camacho and G. Fabbri, Spatial dynamics and convergence: the spatial AK model. J. Econ. Theory 148 (2013) 2719-2736

[26] R. Boucekkine, C. Camacho and G. Fabbri, On the optimal control of some parabolic partial differential equations arising in economics (Special issue in honor of Vladimir Veliov). Serdica Math. J. 39 (2013) 1001-1024

[27] R. Boucekkine, G. Fabbri and F. Gozzi, Egalitarianism under population change: age structure does matter. J. Math. Econ. 55 (2014) 86-100

[28] R. Boucekkine, G. Fabbri, S. Federico and F. Gozzi, Geographic environmental Kuznets curves: the optimal growth linearquadratic case. In: Special issue Economics and the environment: distributed optimal control models. MMNP 14 (2019) 105

[29] R. Boucekkine, G. Fabbri, S. Federico and F. Gozzi, Growth and agglomeration in the heterogeneous space: a generalized AK approach. To appear in: J. Econ. Geogr. (2018) lby041. Doi: 10.1093/jeg/lby041.

[30] M. Braack, M.F. Quaas, B. Tews and B. Vexler, Optimization of fishing strategies in space and time as a non-convex optimal control problem. J. Optim. Theory Appl. (2018) 1-23

[31] A. Bressan, G.M. Coclite and W. Shen, A multidimensional optimal-harvesting problem with measure-valued solutions. SIAM J. Control Optim. 51 (2013) 1186-1202 
[32] P. Brito, The dynamics of growth and distribution in a spatially heterogeneous world. Working Papers Department of Economics 2004-14. ISEG, University of Lisbon (2004)

[33] W. Brock and A. Xepapadeas, Diffusion-induced instability and pattern formation in infinite horizon recursive optimal control. J. Econ. Dyn. Control 32 (2008) 2745-2787

[34] W. Brock, A. Xepapadeas and A.N. Yannacopoulos, Optimal control in space and time and the management of environmental resources. Annu. Rev. Resour. Econ. 6 (2014) 33-68

[35] M. Brokate, Pontryagin's principle for control problems in age-dependent population dynamics. J. Math. Biol. 23 (1985) 75-101

[36] C. Camacho and A. Pérez-Barahona, Land use dynamics and the environment. J. Econ. Dyn. Control 52 (2015) 96-118

[37] C. Camacho and A. Pérez-Barahona, A model in continuous time and space to study economic migration. In: Special issue Economics and the environment: distributed optimal control models. MMNP 14 (2019) 103

[38] D.A. Carlson, A.B. Haurie and A. Leizarowitz, Infinite Horizon Optimal Control: Deterministic and Stochastic Systems. Springer, Berlin (1991)

[39] C. Choquet, E. Augeraud-Véron and E. Comte, Existence, uniqueness and asymptotic analysis of optimal control problems for a model of groundwater pollution. To appear in: ESAIM: COCV (2018). DOI: 10.1051/cocv/2018041.

[40] G.M. Coclite and M. Garavello, A time-dependent optimal harvesting problem with measure-valued solutions. SIAM J. Control Optim. 55 (2017) 913-935

[41] J. de Frutos and G. Martín-Herrán, Spatial effects and strategic behavior in a multiregional transboundary pollution dynamic game. J. Environ. Econ. Manag. (2017)

[42] J. de Frutos and G. Martín-Herrán, Spatial vs. non-spatial transboundary pollution control in a class of cooperative and non-cooperative dynamic games. To appear in: Eur. J. Oper. Res. (2017). Doi: 10.1016/j.jeem.2017.08.001.

[43] G. Fabbri, Geographical structure and convergence: a note on geometry in spatial growth models. J. Econ. Theory 162 (2016) $114-136$

[44] G. Fabbri and F. Gozzi, Solving optimal growth models with vintage capital: the dynamic programming approach. J. Econ. Theory 143 (2008) 331-373

[45] G. Fabbri, F. Gozzi and A. Swiech, Stochastic Optimal Control in Infinite Dimension: Probability and Stochastic Modelling. Springer, Switzerland (2017)

[46] H.O. Fattorini, Infinite Dimensional Optimization and Control Theory. Cambridge University Press, Cambridge (1999)

[47] G. Feichtinger, G. Tragler and V.M. Veliov, Optimality conditions for age-structured control systems. J. Math. Anal. Appl. 288 (2003) 47-68

[48] G. Feichtinger, Ts. Tsachev and V.M. Veliov, Maximum principle for age and duration structured systems: a tool for optimal prevention and treatment of HIV. Math. Popul. Stud. 11 (2004) 3-28

[49] G. Feichtinger and V.M. Veliov, On a distributed control problem arising in dynamic optimization of a fixed-size population. SIAM J. Optim. 18 (2007) 980-1003

[50] M. Fujita and J. Thisse, Economics of Agglomeration. Cambridge University Press, Cambridge (2013)

[51] P. Golubtsov and S.I. Steinshamn, Analytical and numerical investigation of optimal harvesting with a continuously agestructured model. Ecol. Modell. 392 (2019) 67-81

[52] D. Grass, J.P. Caulkins, G. Feichtinger, G. Tragler and D.A. Behrens, Optimal Control of Nonlinear Processes, with Applications in Drugs, Corruption and Terror. Springer, Berlin (2011)

[53] D. Grass and H. Uecker, Optimal management and spatial patterns in a distributed shallow lake model. Electron. J. Differ. Equ. 2017 (2017) 1-21

[54] D. Grass, H. Uecker and T. Upmann, Optimal Fishery with Coastal Catch (2018).

[55] H. Halkin, Necessary conditions for optimal control problems with infinite horizons. Econometrica 42 (1974) $267-272$

[56] Z.R. He and R. Liu, Theory of optimal harvesting for a nonlinear size-structured population in periodic environments. Int. J. Biomath. 7 (2014) 1-18

[57] H.R. Joshi, G.E. Herrera, S. Lenhart and M.G. Neubert. Optimal dynamic harvest of a mobile renewable resource. Nat. Resour. Model. 22 (2009) 322-343

[58] I. Kan, A. Leizerovitz and Y. Tsur, Dynamic-spatial management of coastal aquifers. Optim. Control Appl. Meth. 31 (2009) $29-41$

[59] M.R. Kelly, Jr. Y. Xing and S. Lenhart, Optimal fish harvesting for a population modeled by a nonlinear parabolic partial differential equation. Nat. Res. Model. 29 (2016) 36-70

[60] P. Landi, C. Hui and U. Dieckmann, Fisheries-induced disruptive selection. J. Theor. Biol. 365 (2015) $204-216$

[61] D. La Torre, D. Liuzzi and S. Marsiglio, The optimal population size under pollution and migration externalities: a spatial control approach. A model in continuous time and space to study economic migration. In: Special issue Economics and the environment: distributed optimal control models. MMNP 14 (2019) 104

[62] X. Li and J. Yong, Optimal Control Theory for Infinite Dimensional Systems. Birkhäuser, Boston (1995)

[63] Z. Luo, W.T. Li and M. Wang, Optimal harvesting control problem for linear periodic age-dependent population dynamics. Appl. Math. Comput. 151 (2004) 789-800

[64] F. Mariani, A. Pérez-Barahona and N. Raffin, Life expectancy and the environment. J. Econ. Dyn. Control 34 (2010) 798-815

[65] P. Michel, On the trasversality condition in infinite horizon optimal problems. Econometrica 50 (1982) 975-985

[66] P. Mossay, Increasing returns and heterogeneity in a spatial economy. Reg. Sci. Urban Econ. 33 (2003) 419-444 
[67] C. Simon, B. Skritek and V.M. Veliov, Optimal immigration age-patterns in populations of fixed size. J. Math. Anal. Appl. 405 (2013) 71-89

[68] B. Skritek and V.M. Veliov, On the infinite-horizon optimal control of age-structured systems. J. Optim. Theory Appl. 167 (2015) 243-271

[69] O. Tahvonen, Optimal harvesting of age-structured fish populations. Mar. Resour. Econ. 24 (2009) 147-169

[70] N. Tauchnitz, The Pontryagin maximum principle for nonlinear optimal control problems with infinite horizon. J. Optim. Theory Appl. 167 (2015) 27-48

[71] F. Tröltzsch, Optimal Control of Partial Differential Equations, Vol. 112 of Graduate Studies in Mathematics. AMS, RI (2010)

[72] H. Uecker, Optimal harvesting and spatial patterns in a semiarid vegetation system. Nat. Resour. Model. 29 (2016) 229-258

[73] V.M. Veliov, Optimal control of heterogeneous systems: basic theory. J. Math. Anal. Appl. 346 (2008) 227-242

[74] G.F. Webb, Theory of Nonlinear Age-Dependent Population Dynamics. Marcel Dekker, New York (1985) 